\section{Comment}

The results have both service and theoretical importance. In terms of service developments there have been few reports of self-referrals to psychiatric services in the UK. The opening up of secondary services to self-referrals is a source of anxiety and uniformed speculation. The availability of data on service usage provides a basis from which to inform those involved in planning.

In addition, the differences found between GP and self-referrals allow some speculations to be made about the determinants of self-referral. It must be noted that the data presented have compared one set of secondary service users to another, the difference between these sets being that one has by-passed while the other has been filtered by the GP. This means that the comparisons can show only relative differences, thus making interpretation difficult. They do however, point to two areas of potential interest: sex and social class. While there is a wealth of data published on the use of general practice services by different demographic groups (e.g. Crombie, 1987; Collins \& Klein, 1980), little is known about self-referral to secondary services in the UK. From a theoretical perspective the data presented here provide indicators on which hypotheses and future studies can be based. In this way the monitoring and evaluation of services can be used, not only to provide an overview of service usage but also to promote theoretical investigation.

\section{Acknowledgements}

We would like to thank John Cundy for his assistance with the computer analysis. The study was supported by a grant from the Sainsbury Family Charitable Foundation.

\section{References}

Boardman, A. P. \& Bouras, N. (1988) The Mental Health Advice Centre in Lewisham. Health Trends, 20, 59-63.

Collins, E. \& KLEN, R. (1980) Equity and the NHS: selfreported morbidity, access, and primary care. British Medical Journal, 281, 1111-1115.

Crombre, D. L. (1987) Social Class and Health Status. Inequality or Difference? Royal College of General Practitioners Occasional Paper 25. Exeter: RCGP.

GOLDBerg, D. \& HuXuEY, P. (1980) Mental Illness in the Community. The Pathway to Psychiatric Care. London: Tavistock.

Hutron, H. (1985) Self-referrals to a community mental health centre: A three year study. British Journal of Psychiatry. 147, 540-544.

Lm, M. H. (1983) A psychiatric emergency clinic: A study of attendences over six months. British Journal of Psychiatry, 143, 460-466.

MCAusland, T. (1985) Planning and Monitoring Community Mental Health Centres. London: Kings Fund Centre.

SAYCE, E. (1987) Community Mental Health Centres Report of the Annual Conference 1987. London: NUPRD.

Details of the data are available from the authors on request.

\title{
Quality of life in a residential setting
}

\section{Ian LeONARD, Research Registrar in Psychiatry, The David Lewis Centre for Epilepsy, Near Alderley Edge, Cheshire SK9 7UD}

The David Lewis Centre for Epilepsy has approximately 300 adult residents. All have epilepsy, which is often only partly alleviated by available treatments; many have mental handicap or psychiatric illness, adding to their total disability. The Centre's explicit aim of understanding and caring for people with epilepsy implies concern not just for clinical measures such as seizure frequency, but for our residents' quality of life.

Two trends in patient management over recent years combine to focus interest on quality of life measurement in residential settings. One is the shift to community from residential care, too often with an untested assumption of the former's intrinsic super- iority. The other is the increasing recognition of the necessity of service evaluation to ensure the optimal use of finite resources and justify the continuation of those resources.

While the need for evaluation of quality of life in institutions is clear, the form that evaluation should take is not. Symptoms and their alleviation seem relevant, yet this is insufficient when institutions impinge on all areas of their residents' lives. Their physical surroundings, activities through the day and social milieu are just a few examples of spheres where such influence is marked. Day \& Klein (1987) reviewed the assessment of quality of care and suggested that in the difficult area of outcome 
measurement it is prudent to concentrate on the avoidance of negative occurrences - bed sores are easier to count than self esteem. Without doubt bad institutional practices and abused residents do exist and the Whittingham inquiry (1972) is too contemporary for complacency. Nonetheless it is important to focus on more than the avoidance of harm.

The problem of which aspects of life to measure still remains however. There is no single assessment acknowledged as a "gold standard" (Spitzer, 1987) and various published scales include frequency of vomiting, having one hot meal per day and driving a car. None of these would be a sensitive index for our population. Many quality of life scales appear to have had their content decided by decree of the investigators, although one well validated measure (Spitzer et al, 1981) had its content decided by committees of patients, relatives and health workers. This paper reports on a study of the important components of quality of life as seen by residents, their relations and staff at the David Lewis Centre.

\section{The study}

A 50 item questionnaire was devised where each subject was requested to rate how important (essential, fairly important, not very. important or not at all important) each item was for a resident's quality of life. The questionnaire was distributed to all staff plus relations who visited over a two week period. A random sample of residents were given a semistructured interview based on the questionnaire.

The inclusion of particular items was based on a review of the literature and informal discussions with residents, relations and staff. Examples of these used were - warm dry accommodation, close staff supervision, being in good spirits and opportunity to attend events outside the Centre.

The data were analysed in two ways. First the items were ranked in order of perceived importance for each group. In addition the relative importance of each item to the three groups was examined (contingency table groups $\mathrm{X}$ relative importance, Chisquared test). All 50 items were so assessed and a statistical significance level of $\boldsymbol{P}<0.01$ used. Reference to inter-group difference subsequently implies this level of significance.

\section{Findings}

Thirty-five residents were interviewed to obtain 25 satisfactory assessments, ten were unable to take part due to their degree of dementia or mental handicap.

One hundred and sixty-seven staff received the questionnaire and 135 were returned, a response rate of $81 \%$ : 25 relations also completed the questionnaire.

A broad pattern emerged in the ranking of the items. This same pattern was discernible in each group separately. Those which concerned basic physical needs such as warm, dry accommodation, an adequate diet and clothing along with the availability of medical and nursing care were ranked most highly.

A little less importance was attached to having as few seizures as possible plus a group of items reflecting independence - personal possessions, the opportunity for privacy and being ambulant are examples.

Thereafter came items which concerned social relationships (the availability of close friends and facilities to encourage socialising) mixed with those related to occupation (the availability of and taking part in work and hobbies).

Items reflecting more abstract concepts such as intellectual and creative needs were generally ranked least highly.

Despite the agreement between groups on the general areas of importance, more detailed analysis of individual items did reveal interesting differences in emphasis. Relations attached significantly more importance to close supervision by regular staff in a settled environment than did staff and much more than did residents. Conversely, residents placed significantly greater importance on being in good spirits. Indeed, they ranked it as highly as basic physical needs. Residents also placed significantly greater importance on being able to get out of the Centre whether for shopping, day trips or just a change of scene. In addition, they gave significantly more importance to the need for close friends and opportunity for sexual relationships. This may have contributed to the greater importance they also attached to having their own single bedroom.

\section{Comments}

A moderate consensus has been demonstrated on the relative importance of the constituents of quality of life for our residents. The order in which items were placed - physical needs, independence, social and occupational activities followed by more abstract needs - is reminiscent of Maslow's hierarchy (1943). His examination of human motivation produced a hierarchy of needs from the physiological through safety, love and esteem to self actualisation. This scheme requires the satisfaction of the more basic needs before the next can be addressed. In this sense they are more important.

Symptoms and their alleviation were placed just below basic physical needs in importance by all the study groups. This reflects how epilepsy, if poorly controlled, can affect a wide range of functioning adversely. Nonetheless, it cannot be assumed that the treatment of all illness would be attributed this level of importance in every setting. For example, here psychological well-being was placed very highly by residents, but not other groups. 
Of the important categories identified, basic physical needs is the one most often neglected by the available quality of life measures. There is a frequent assumption that shelter and food are adequately provided, although Jones (1985) in a study of discharged previously long-stay psychiatric in-patients has shown that this is not wholly warranted.

Along with the measurement of the provision for basic physical needs, separate measures of symptoms, independence, social relationships and how time is occupied are shown to be important by this study. Certainly they must be included in any assessment which purports to examine quality of life comprehensively. Symptoms may be disease-specific as in seizure control but an index of psychological morbidity should also be incorporated because of its importance to the resident group. Conveniently, abstract concepts such as intellectual satisfaction and artistic achievement which are the most diffcult to measure were also seen as of lesser importance.

An argument must also be made for each area to be considered separately and no overall scores derived. The loss of information so created is major and the constituents of quality of life do not necessarily covary. Indeed improvement in one area may be gained by reduction in another, for example ensuring basic physical needs may restrict independence.

With respect to selecting appropriate instruments, an example relevant to this study is that the importance of time spent away from the Centre by residents was highlighted. For our population any assessments need to be observer-rated and easily completed by a variety of staff. This is fulfilled by the Index of Community Involvement for Individuals developed and used by Raynes \& Sumpton (1987) to assess the quality of residential provision for mentally handicapped people.

The extent of residents' participation in life outside the Centre was one of the issues they viewed as more important than other groups. The level of such activity has increased at this institution and doubtless many others, over recent years, but difficulties remain. One factor in this is the geographic isolation of many institutions combined with a lack of public transport. Thus residents with relevant abilities may still be unable to go out as they wish. Arrangements to take out those without such abilities are inevitably labour-intensive, either of staff or volunteers, and therefore relatively infrequent.

A broader difference was over the greater emphasis placed on stability and close supervision by relations than residents, with staff views being intermediate. Essentially residents see stability as boring. Staff changes and movement between houses were generally seen as providing relief from what is too often an unstimulating environment. A corollary to this is that if residents are or become unable to express their needs then their relations may well be inaccurate advocates for them.

The greater importance attached to the opportunity for sexual relationships by residents was not unexpected. However, their views here also demonstrate how individuals vary in the detail of what is central to their quality of life. The resident sample included one partner from a couple with a long-term relationship who clearly desired but lacked a private space to pursue a sexual relationship. Others viewed sex as an irrelevance because of lack of personal interest or the single status of nearly all the residents implying immorality of any sexual relationships. As noted, earlier institutions do influence all areas of their residents' lives and the limitation of privacy is of particular importance here. There is a tradition of ignoring this field of activity in long-stay institutions but there are reasons to change this. If concern for independence and quality of life does not make an impact then the increasing prevalence of HIV infection may do so.

\section{Concluding remarks}

This study has identified a consensus over the relative importance of the constituents of quality of life in institution. The difference of priorities in some areas shown by residents, however, must be noted as it is their quality of life which must be the raison d'être of such establishments. Relative scarcity of resources or our own moral views should not be allowed to prevent issues being considered.

\section{Acknowledgement}

I would like to thank all the residents, staff and relatives who completed the questionnaire and $\mathrm{Dr}$ Stephen Brown for his help and encouragement.

\section{References}

DAY, P. \& KLEIN, R. (1987) Quality of institutional care and the elderly: policy issues and options. British Medical Journal, 294, 384-387.

JONES, K. (1985) After Hospital: A Study of Long-term Psychiatric Patients in York. Department of Social Policy and Social Work, University of York.

MasLow, A. H. (1943) A theory of human motivation. Psychological Review, L, 270-296.

RAYNES, N. V. \& SUMPTON, R. C. (1987) Differences in the quality of residential provision for mentally handicapped people. Psychological Medicine, 17, 999-1008.

Report of the Committee of Inquiry into Whittingham Hospital (1972). London: HMSO.

SPITZER, W. O. (1987) State of science 1986: Quality of life and functional status as target variables for research. Journal of Chronic Diseases, 40, 465-471.

-, Dobson, A. J., Hall, J., Chesterman, E., LeVI, J., SHEPHERD, R., BATtista, R. N. \& CATChLOVE, B. R. (1981) Measuring the quality of life of cancer patients. Journal of Chronic Disease, 34, 585-597. 